\title{
Silicon-on-insulator tunable waveguide-coupled microdisk resonators with selectively integrated $p$-i-n diodes
}

\author{
Linjie Zhou and Andrew W. Poon \\ Department of Electrical and Electronic Engineering, The Hong Kong University of Science and Technology, \\ Clear Water Bay, Hong Kong SAR, China \\ Tel: (852)-2358-7905; Fax:(852)-2358-1485; Email: eeawpoon@ust.hk
}

\begin{abstract}
We report a design and analysis of compact silicon-on-insulator tunable waveguide-coupled microdisk resonators with selectively integrated p-i-n diodes. Our simulations suggest that singlemode resonances can be coupled and blueshifted by free-carrier plasma dispersion effect.

(C)2005 Optical Society of America
\end{abstract}

OCIS codes: (230.5750) Resonators; (230.2090) Electro-optical devices

Silicon-based active devices such as tunable filters, switches and modulators using free-carrier plasma dispersion effect have long been attracting considerable research interest [1]. However, typical refractive index change $\Delta \mathrm{n}$ demonstrated is only in the order of $10^{-3}$, which imposes relatively long mm- to cm-sized phase-shifters in conventional Mach-Zehnder interferometer designs [1,2]. In order to attain compact active silicon devices that are sensitive to small $\Delta \mathrm{n}$, Barrios et al. recently proposed and demonstrated compact silicon-on-insulator (SOI) tunable Fabry-Perot microresonators using Bragg mirrors with laterally integrated p-i-n diodes [3].

In this summary, we propose and analyze compact SOI tunable laterally waveguide-coupled microdisk resonators with selectively integrated lateral p-i-n diodes. We also report initial experimental results of SOI passive laterally waveguide-coupled microdisk resonators as a necessary step towards active SOI microdisk devices.

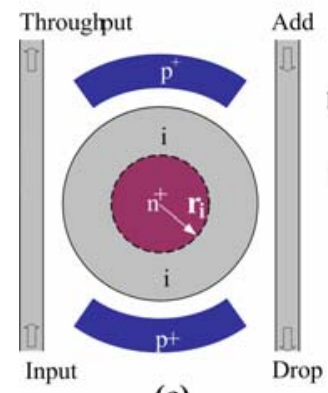

(a)

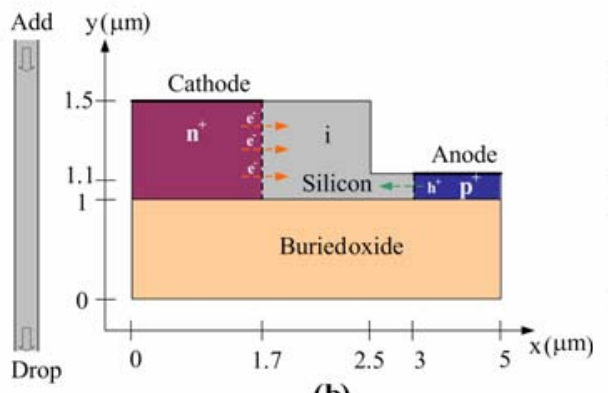

(b)
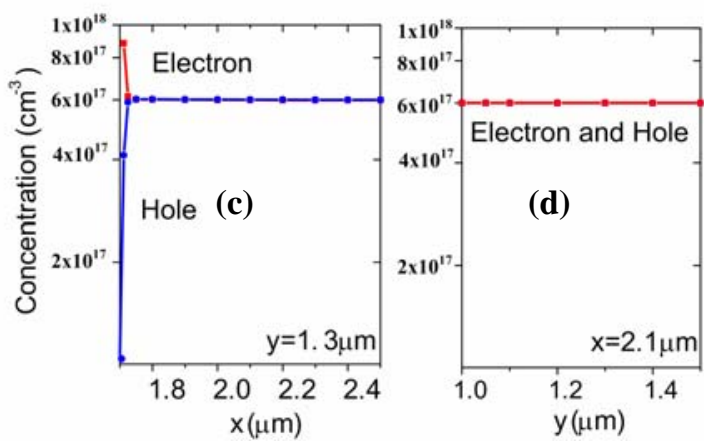

Fig.1. (a) Schematic of a SOI waveguide-coupled microdisk resonator with selectively integrated lateral p-i-n diodes as a tunable channel add-drop filter. (b) Schematic cross-section of the lateral p-i-n diode (under forward bias). (c) Horizontal distributions of free-carriers concentration along $y=1.3 \mu \mathrm{m}$ in the intrinsic region. (d) Vertical distributions of free-carriers concentration along $\mathrm{x}=2.1 \mu \mathrm{m}$ in the intrinsic region.

Figure 1 (a) shows the schematic of an active microdisk resonator side-coupled with submicrometer single-mode input and output-coupled waveguides. We use lateral $\mathrm{p}$-i-n diodes with $\mathrm{n}^{+}$-doped region in the microdisk central region and $\mathrm{p}^{+}$-doped region outside the microdisk rim. Upon forward bias, free-carriers are 
selectively injected into the arc intrinsic region with lateral p-i-n diodes. The arc region refractive index and absorption can then be selectively tuned by means of free-carrier plasma dispersion effect. Doping can be made by implantation in order to precisely control the lateral dopant profiles. Figure 1 (b) shows the schematic cross-sectional view of our lateral p-i-n diode.

We used semiconductor device simulator MEDICI to simulate the free-carriers concentration profiles. We assumed a uniform dopant concentration of $10^{20} \mathrm{~cm}^{-3}$ for the $\mathrm{n}^{+}$region and of $10^{19} \mathrm{~cm}^{-3}$ for the $\mathrm{p}^{+}$region. Figures 1 (c) and 1 (d) show the calculated electron and hole-carrier distributions by applying a forward bias voltage of $0.91 \mathrm{~V}$ at $\mathrm{y}=1.3 \mu \mathrm{m}$ and $\mathrm{x}=2.1 \mu \mathrm{m}$ in the intrinsic region. We observed a flat distribution of about $6 \times 10^{17} \mathrm{~cm}^{-3}$ for both carriers. Using the free-carrier dispersion at a wavelength of $1550 \mathrm{~nm}$ [4], we calculated the refractive index change $\Delta \mathrm{n}=-0.002$ and the associated free-carrier absorption coefficient change $\Delta \alpha=8.7 \mathrm{~cm}^{-1}$ at the intrinsic region, and a substantial $\Delta \alpha=850 \mathrm{~cm}^{-1}$ at the central $\mathrm{n}^{+}$region. Hence, by selectively integrating the lateral p-i-n diodes, we achieve two objectives - (i) preferentially inject carriers to the intrinsic rim region to phase-shift the low-order whispering-gallery modes (WGMs), and (ii) suppress the undesirable high-order WGMs by excessive free-carrier absorption in the microdisk central region.

We used two-dimensional finite-difference time-domain (FDTD) simulations to demonstrate the suppression of high-order WGMs by tuning the size of the heavily doped central region. Here we fix the $\mathrm{n}^{+}$-concentration at $10^{20}$ $\mathrm{cm}^{-3}$. We simulated a 5- $\mu \mathrm{m}$ diameter microdisk resonator. Figures 2 (a) and 2 (b) show the FDTD simulated transmission spectra with the $\mathrm{n}^{+}$disk radius $\mathrm{r}$ of $1.2 \mu \mathrm{m}$ and $1.7 \mu \mathrm{m}$. With an increase in the central $\mathrm{n}^{+}$doped area, the low-order mode A is only slightly shifted (with essentially fixed Q and coupling efficiency), whereas the high-order mode B is partially suppressed. Figure 2 (c) shows the simulated steady-state mode-field patterns of modes A' and B' with the $\mathrm{r}=1.7 \mu \mathrm{m} \mathrm{n}^{+}$doped region.
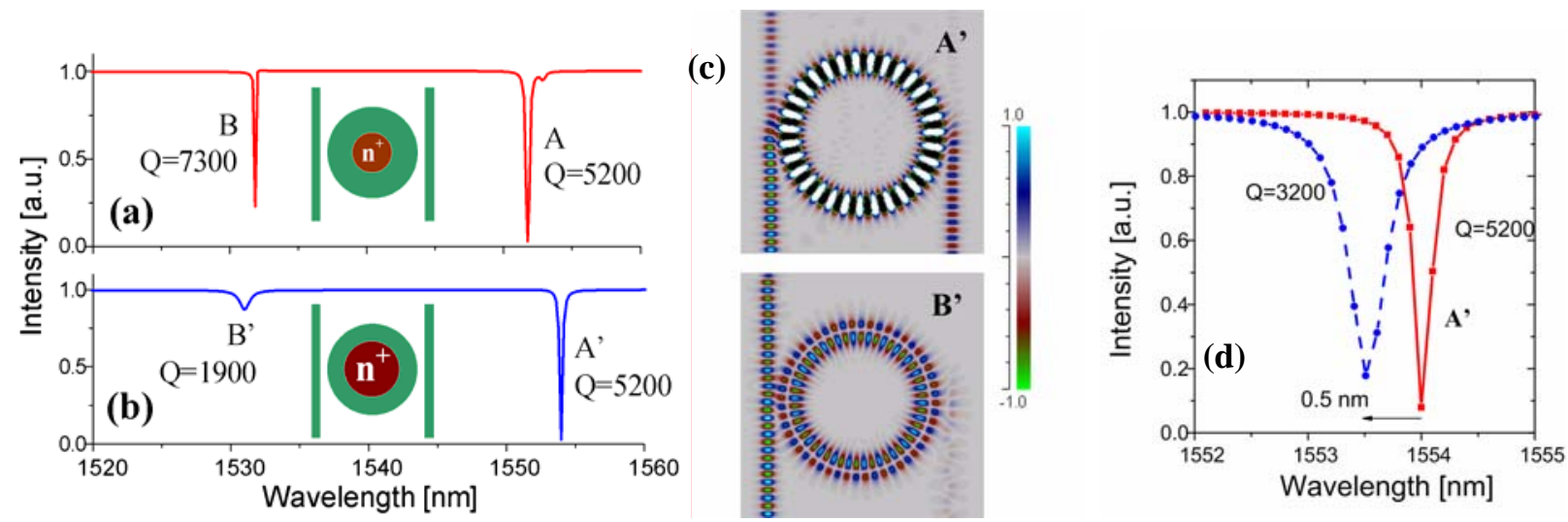

Fig.2. FDTD-simulated transmission spectra at throughput port for 5- $\mu \mathrm{m}$-diameter microdisk resonators (TM polarized). (a) $\mathrm{n}^{+}$doped disk radius $\mathrm{r}=1.2 \mu \mathrm{m}$, and (b) $\mathrm{r}=1.7 \mu \mathrm{m}$. We adopted the waveguide refractive index $\mathrm{n}=3.45$, and the background refractive index of 1.45. (c) Simulated steady-state mode-field patterns of the low-order mode A' and of the partially suppressed high-order mode B'. (d) Simulated mode A' transmission when there is no bias (solid line) and the blueshifted resonance transmission upon a forward biasing of $0.91 \mathrm{~V}$ (dashed line).

Figure 2 (d) shows the FDTD-simulated mode A' resonance blueshift of about $0.5 \mathrm{~nm}$ upon a forward bias of $0.91 \mathrm{~V}$ by imposing a $\Delta \mathrm{n}=-0.002$ and $\Delta \alpha=8.7 \mathrm{~cm}^{-1}$ along the arc region with carrier injection. Here we assumed the total active arc length is one-half of the total microdisk circumference (Fig.1(a)). When there is no bias (solid line), the resonance has a $\mathrm{Q}=5,200$ and a coupling efficiency of about $92 \%$. Upon forward bias (dashed line), the blueshifted resonance has a reduced $\mathrm{Q}=3,200$ and a reduced coupling efficiency of about $85 \%$. 
As a necessary step towards active SOI microdisk devices, we demonstrate for the first time to our knowledge SOI passive laterally waveguide-coupled microdisk resonators. We employed standard silicon microfabrication processes. Figure 3 (a) shows the scanning-electron micrograph of our initially fabricated waveguide-coupled $50-\mu \mathrm{m}$-diameter passive microdisk resonator on $0.5-\mu \mathrm{m}$ SOI substrates. Inset shows the cross-sectional view of the side-coupled submicrometer singlemode waveguide with a waveguide height of about $0.35 \mu \mathrm{m}$ and a width of about $0.35 \mu \mathrm{m}$. Figure 3 (b) shows the measured multimode throughput transmission spectrum with TE polarization. Figure 3 (c) shows a zoom-in view of a high-Q mode $(\mathrm{Q} \approx 32,000)$ with exceeding $16 \mathrm{~dB}$ coupling efficiency. This high-Q value is an order of magnitude higher than that recently reported in SOI waveguide-coupled microring resonator [5].
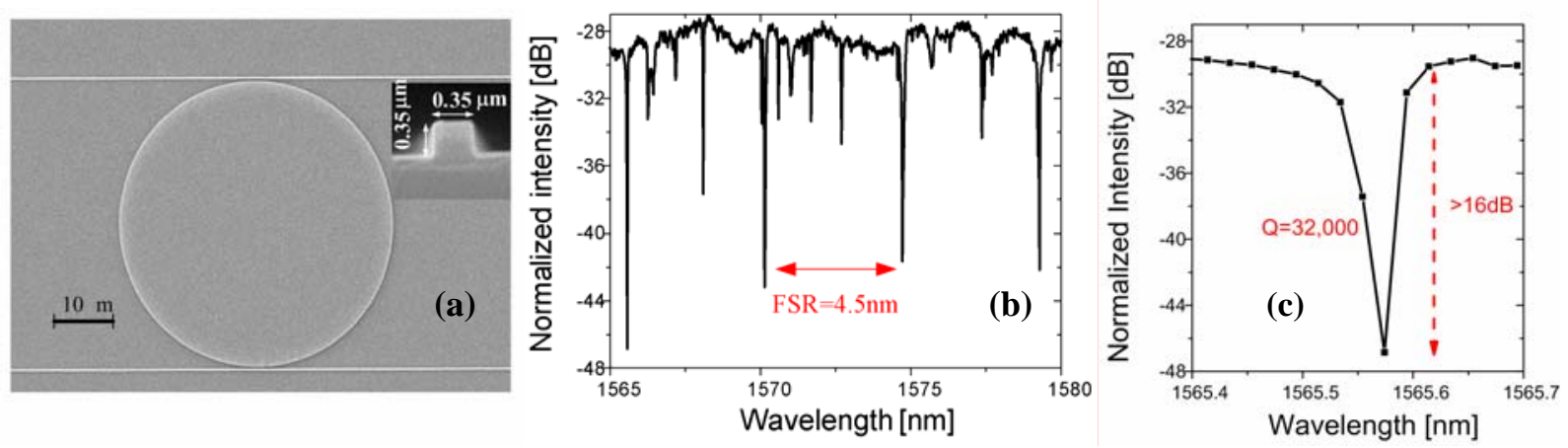

Fig.3. (a) Scanning-electron micrograph of SOI passive waveguide-coupled microdisk resonator on $0.5 \mu \mathrm{m}$ SOI. Inset shows the cross-sectional view of the submicrometer singlemode waveguide. (b) Measured multimode throughput transmission spectrum with TE polarization. The free-spectral range (FSR) of $4.5 \mathrm{~nm}$ is consistent with the microdisk circumference. (c) Zoom-in view shows the high-Q mode with $\mathrm{Q} \approx 32,000$ and a coupling efficiency > $16 \mathrm{~dB}$.

In summary, we report a design and analysis of injection-type active microdisk resonator devices using selectively integrated lateral p-i-n diodes. We took advantage of the large optical absorption loss in the heavily doped microdisk central region in order to suppress undesirable high-order whispering-gallery modes. While the lateral p-i-n diodes selectively inject carriers to phase-shift the low-order whispering-gallery modes along the microdisk rim. Our simulations suggested a resonance wavelength blueshift of about $0.5 \mathrm{~nm}$ under a forward bias of only $0.91 \mathrm{~V}$. We also experimentally demonstrated for the first time to our knowledge SOI passive waveguide-coupled microdisk resonators with a high Q of about 32,000 and a high coupling efficiency exceeding 16 $\mathrm{dB}$. Design optimization and fabrication of the proposed SOI active microdisk devices using standard silicon microfabrication processes are in progress.

\section{References}

[1] G T Reed and A P Knights, Silicon Photonics: An Introduction (John Wiley and Sons, 2004).

[2] A. Liu, R. Jones, L. Liao, D. Samara-Rubio, D. Rubin, O. Cohen, R. Nicolaescu, M. Paniccia, “A high-speed silicon optical modulator based on a metal-oxide-semiconductor capacitor”, Nature 427, 615-618 (2004).

[3] C. Angulo Barrios, V. R. Almeida, R. R. Panepucci, B. S. Schmidt, and M. Lipson, “Compact silicon tunable Fabry-perot resonator with low power comsumption”, IEEE Photon. Technol. Lett. 16, 506-508 (2004).

[4] R. A. Soref and B. R. Bennett, “Electrooptical effects in silicon”, IEEE J. Quant. Electron 23, 123-129 (1987).

[5] P. Dumon, W. Bogaerts, V. Wiaux, J. Wouters, S. Beckx, J. V. Campenhout, D. Taillaert, B. Luyssaert, P. Bienstman, D. V. Thourhout, and R. Baets, “Low-loss SOI photonic wires and ring resonators fabricated with deep UV lithography”, IEEE Photon. Technol. Lett. 16, 1328-1330 (2004). 